\title{
Optimisation and failure criteria for composite materials by the boundary element method
}

\author{
S. Debbaghi*, A. Sahli**, Sara Sahli*** \\ *Laboratoire de Mécanique Appliquée, Département de Mécanique, Université des Sciences et de la Technologie d'Oran \\ USTO MB, B.P. 1505 El M'Naouer, 31000, Oran Algérie, E-mail: mechanics153@yahoo.com \\ **Université Ibn Khaldoun de Tiaret, E-mail: sahli_ah@yahoo.com \\ ***Université d'Oran \\ cross $^{\text {ref }}$ http://dx.doi.org/10.5755/j01.mech.23.4.14881
}

\section{Introduction}

With the increasing number of projects with composite materials came the need to calculate stresses and strains in material structures anisotropic. This task is more complicated than in isotropic materials, since the anisotropy increases the number of variables of the problem. Analytical solutions for anisotropic problems are restricted to a small number of simple field's problems. In the case of structures and mechanical components, the field becomes quite complex, and its only possible through the analysis and numerical methods or experimental methods. With the evolution of computers numerical methods began to be used for a much wider range of problems. Among the numerical methods that stood out in treatment of structural problems are the method of boundary elements and the method of finite elements. Although obtaining a formulation of boundary elements is mathematically harder than the finite elements, the boundary elements have very desirable characteristics for modeling many problems such as:

- They can model and problems of high gradient of tension and deformation and

- only the contour is discretized. It has recently increased significantly the number of tasks where the boundary element method has been used in the analysis of problems involving composite materials and anisotropy [1-9].

Structural optimization has seen accelerated deployment throughout all industries in the past decade, largely due to the recognition that tremendous efficiency gain can be achieved at concept design stage through topology optimization (see, e.g., [10-13] for early contributions and surveys). For metal structure, a two phase design process has become well established, where at Phase-I topology optimization is applied to generalize design concept, while design details are further optimized using sizing and shape optimization at Phase-II [14-18]. For composite structure, the added design freedom prompts a modification of the process leading from concept to design details. While different forms of composite materials exist, the predominant usage is composite laminate where thin plies of various orientations are stacked together to form a shell structure. In recent years, the authors have developed a Three-Phase optimization process for composite laminate design optimization [19-21].

The target of the first phase is the material distribution in terms of orientation and thickness. This is achieved through free-size optimization where thickness of each 'super-ply' of a unique fiber direction is allowed to change freely throughout the structure. As a result thickness contour of each fiber orientation is obtained. A discrete interpretation of the thickness contour results in concept design of ply layout and thickness. Then in Phase-II the interpreted ply-based structural model is further optimization under all design constraints with discrete design variables representing the number of plies of each ply patch. During Phase-III, ply stacking optimization is performed to refine the design according to detailed manufacturing constraints. It should be emphasized that manufacturing constraints are considered throughout all three optimization phases. For example, one important design/manufacturing requirement of aerospace OEMs is that no plies of same orientation is allowed to be stacked continuously for more than 3 or 4 plies. Such requirement would translate into percentage requirement during Phase-I and II so that a balanced distribution of fiber orientation is achieved to allow feasible stacking during Phase-III.

Farshi and Herasati [22] presented a method for optimization of weight blades of fibrous composite materials under action of lateral loads.

The purpose, in this paper, is the laminate design composite materials and minimum thickness that can support multiple static loads applied in the normal direction to the surface without the occurrence of failures in any of the layers, according to the criterion of Tsai-Hill. In this investigation, the orientation angles of the fibers is treated as discrete variables, which can vary only by a pre-designated increase, while the thicknesses of the layers are treated as continuous variables.

The optimization procedure is a strategy based on two stages: the first only the orientation angles of the fibers of the layers is treated as design variables, and second, only the thickness of the layers.

\section{Failure criteria for composites materials}

The theories of failure are central to the determination of criteria for the prediction of failure of a material against a bi-or three-dimensional state tensions. When the state of tensions is one-dimensional, the simple criterion to control the amount of tension so as not to exceed the yield stress or rupture of the material is sufficient to determine fault. However, a complex state of tensions requires own theories for the type of material.

Looking at the data presented it follows easily that the failure in composite materials and more complex than in isotropic materials, it involves the analysis of a larger number of variables. Thus, the criteria traditionally used in the design of machines and structures in materials with isotropic 
behavior may not be appropriate and may even lead the designer to catastrophic errors if used wrongly.

The utilization of more complex criteria leads to more accurate results, but at a higher cost. The criteria of maximum stress and maximum deformation require the analysis of three different fault conditions for the determination of the actual limit. This inconvenient is eliminated with the development of quadratic criteria such as Tsai Hill, Hill [23], Tsai [24], and Tsai - Wu [25]. While the first Tsai - Hill is simpler to use the second Tsai-Wu presents a better accuracy of the results. All these criteria however require a larger number of tests of composite materials (unidirectional laminate) than those required for determining the characteristics of an isotropic material.

The formulation developed in this paper is applied to compute the stress of plane structures of composite laminates subjected to traction loads to the structure plane. Will be studied in this work the failure criteria for composite materials, focusing on the quadratic criterion of Tsai-Hill and Tsai-Wu [25].

\section{Basic relations for anisotropic elasticity}

Using the notation reduced tensor proposed by Lekhnitskii [26], the equation for anisotropic elasticity may be written as:

$$
a_{11} \mu^{4}-2 a_{16} \mu^{3}+\left(2 a_{12}+a_{66}\right) \mu^{2}-2 a_{26} \mu+a_{22}=0,
$$

where $a_{i j}$ is the material compliance matrix given by [26]:

$$
\begin{aligned}
& a_{11}=\frac{1}{E_{1}}, \quad a_{12}=-\frac{v_{12}}{E_{1}}=-\frac{v_{21}}{E_{2}}, \quad a_{16}=\frac{\eta_{12,1}}{E_{1}}=\frac{\eta_{1,12}}{G_{12}}, \\
& a_{22}=\frac{1}{E_{2}}, \quad a_{26}=\frac{\eta_{12,2}}{E_{2}}=\frac{\eta_{2,12}}{G_{12}}, \quad a_{66}=\frac{1}{G_{12}},
\end{aligned}
$$

where $E_{k}$ are Young's moduli referring to axes $x_{k}, G_{12}$ is the shear modulus for the plane, $v_{i j}$ are Poisson's ratios and $\eta_{j k, l}$ and $\eta_{l, j k}$ are mutual coefficients of the first and second kind, respectively, and $\mu$ the roots of the equation, always complex or pure imaginary, occurring in pairs $\left(\mu_{\mathrm{k}}\right.$ and $\left.\bar{\mu}_{\mathrm{k}}\right)$ as shown by Lekhnitskii [27].

\section{Boundary integral equation for anisotropic materials}

The integral equation, which relates the fundamental state with any other state in a body with domain $\Omega$ and boundary $\Gamma$, can be written for an interior domain point as (see for example Reference [28]):

$$
u_{i}+\int_{\Gamma} T_{i k} u_{k} d \Gamma=\int_{\Gamma} U_{i k} t_{k} d \Gamma,
$$

where $u_{i}$ is the displacement vector, $t_{k}$ is the traction vector, $U_{i k}$ and $T_{i k}$ are the displacement and traction anisotropic fundamental solutions for elastostatics, respectively.

The anisotropic displacement fundamental solution for elastostatics can be written as:

$$
U_{j i}=2 \operatorname{Re}\left[q_{i 1} A_{j 1} \ln \left(z_{1}-z_{1}^{\prime}\right)+q_{i 2} A_{j 2} \ln \left(z_{2}-z_{2}^{\prime}\right)\right],
$$

where $q_{i k}$ is equal to

$$
q_{i k}=\left[\begin{array}{c}
a_{11} \mu^{2}+a_{12}-a_{16} \mu \\
a_{12} \mu+\frac{a_{22}}{\mu}-a_{26}
\end{array}\right],
$$

z, z' the complex variables Defining as [29]:

$$
\begin{aligned}
& z=\left\{\begin{array}{l}
z_{1} \\
z_{2}
\end{array}\right\}=\left\{\begin{array}{l}
x_{1}+\mu_{1} x_{2} \\
x_{1}+\mu_{2} x_{2}
\end{array}\right\}, \\
& z^{\prime}=\left\{\begin{array}{l}
z_{1}^{\prime} \\
z_{2}^{\prime}
\end{array}\right\}=\left\{\begin{array}{l}
x_{1}^{\prime}+\mu_{1} x_{2}^{\prime} \\
x_{1}^{\prime}+\mu_{2} x_{2}^{\prime}
\end{array}\right\},
\end{aligned}
$$

where $x_{1}$ and $x_{2}$ are the field point co-ordinates, $x_{1}^{\prime}$ and $x_{2}^{\prime}$ are the source point co-ordinates.

$A_{i k}$ is the solution vector of the linear system.

\section{Structural optimization of composite materials}

\subsection{Aspects of optimization}

The optimization of techniques is used to find a set of values great for the parameters that can be changed during the project. These parameters are called variables projects and are denoted by a vector $x=\{x 1, x 2, \ldots ., x n\}$. The variables projects can be weight, size, thickness of the layers, the direction of the fibers, etc. These variables project can be continuous or discrete. Continuous variables have a range of variation and can take any value within this range. The variables can only have discrete values isolated, typically from a list of allowed values. The choice of design variables will be able to be decisive for project success. In particular, it is important to make sure that the choice of variables is consistent with the model to be analyzed. The idea of optimization implies the existence of some function $f(x)$ or functions $f(x)=\{f(x 1), f(x 2), \ldots \ldots, f(x n)\}$. can be improved and can be used as measures for the efficiency of the project. These functions are called objective functions. The optimization with more than one objective function is called optimization of multiple criteria. For problems of structural optimization, weight, displacement, stress, frequency of vibrations, costs, or a combination of these can be used as objective function [30].

\subsection{Dimensional optimization: section method}

The Golden Section method estimates the maximum, minimum or zero of a function of a variable. It is a popular technique for several reasons: First, if the function is assumed unimodal, that is, has only one maximum point or minimum in the search range, this need not be derived from continuous [31]. Second, unlike the polynomial techniques or other based on curve fitting, the rate of convergence for the method of golden section is known. The method of golden section is based on the technical limitation of the search to a range, that is, is to reduce the search universe of limits until the interval formed by the boundaries is smaller than a admissible error [32]. 
As in an iterative process, one must set a criterion to identify when the process converges to the acceptable solution. Assuming an initial uncertainty range, $x_{u}-x_{l}$, it is desired to reduce the interval for any $\varepsilon$ initial interval, called the relative tolerance, or for some $\Delta x$ which is an absolute tolerance. The value $\varepsilon$ will be found as follows:

$$
\varepsilon=\frac{\Delta x}{x_{u}-x_{l}}
$$

\section{Numerical results}

\subsection{Circumferential tensions in an infinite plate with hole}

To compare the values obtained for the tensions in the literature, an infinite plate with a circular hole (Fig. 1) has been chosen. An analytical solution to this problem was presented by [26]. Figure 1shows a section of an infinite orthotropic plate with a circular hole, subject to traction loads applied in the direction of the lower modulus of elasticity $E_{2}$. The distribution of circumferential tension over the hole is given by:

$$
\sigma_{\theta \theta}=\sigma \frac{E_{\theta}}{E_{1}} \operatorname{Re}\left\{\mu_{1} \mu_{2}\left[\mu_{1} \mu_{2}+i\left(\mu_{1} \mu_{2}\right) \cos ^{2} \theta+\sin ^{2} \theta\right]\right\},
$$

where $\theta$ is the polar angle measured from the axis $x_{1}$, and $E_{\theta}$ is the Young's modulus in the direction tangent to the contour of the hole which is given by:

$$
E_{\theta}=\left[\frac{\sin ^{4} \theta}{E_{1}}+\left(\frac{1}{G_{12}}-\frac{2 v_{12}}{E_{1}}\right) \sin ^{2} \theta \cos ^{2} \theta+\frac{\cos ^{4} \theta}{E_{2}}\right]^{-1} \text {. }
$$

The distribution of tension given by Eq. (9) is symmetrical in the major axis directions and the maximum tension occurs at points $\mathrm{A}$ and $\mathrm{C}$ in Fig. 1 and are given by:

$$
\sigma_{\theta \theta}^{A}=\sigma_{\theta \theta}^{C}=\sigma \operatorname{Re}\left[1+i\left(\frac{1}{\mu_{1}}+\frac{1}{\mu_{2}}\right)\right] \text {. }
$$

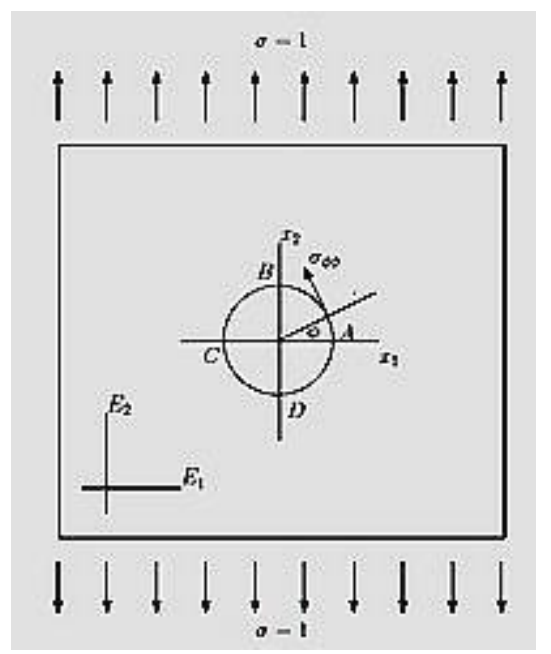

Fig. 1 Circular hole in an infinite orthotropic plate

The minimum tension occurs in B and D in Fig. 1 and are given by:

$$
\sigma_{\theta \theta}^{B}=\sigma_{\theta \theta}^{D}=\sigma \operatorname{Re}\left[\mu_{1} \mu_{2}\right]
$$

This problem was modeled using the formulation of boundary elements presented in this chapter and results were compared with the analytical solution. In modeling, it was considered approximation for the infinite lamina, a lamina with square hole, and the edge of the lamina is 9 times the hole radius size. They were used 46 elements of continuous quadratic contour, 28 in the outer contour and 18 in hole. The material properties were follows: $E_{1}=11,8 \mathrm{GPa}$, $E_{2}=5,89 \mathrm{GPa}, G_{12}=0,69 \mathrm{GPa}$ and $v_{12}=0 ; 071$.

Fig. 2 shows the circumferential tensions calculated by the boundary elements method, compared with the analytical solution given by Eq. (9). As can be noted there is a perfect concordance the results.

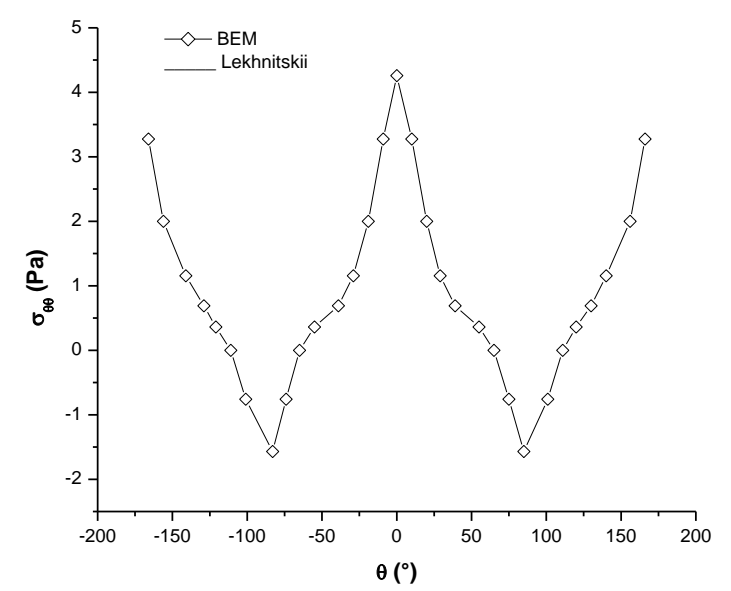

Fig. 2 Calculation of the circumferential stressplate for $\pi \leq \theta \leq \pi$

\subsection{Failure criteria for a plate without hole}

Consider a square lamina in width $=1 \mathrm{~m}$ and thickness $t=1 \mathrm{~mm}$ an orthotropic material that is under traction $\sigma=10 \mathrm{MPa}$, as shown in Fig. 3. The material properties are: $E_{1}=240 \mathrm{GPa}, E_{2}=18,5 \mathrm{GPa}, G_{12}=5,59 \mathrm{GPa}, v_{12}=0 ; 23$. The main axis of the material is oriented of an angle $\theta$ in relation with horizontal. The property resistance of materials are $X_{t}=1260 \mathrm{MPa}, Y_{t}=61 \mathrm{MPa}$ and $S=67 \mathrm{MPa}$. The tensions written in the main reference material when $\theta=45^{\circ}$ are equal to:

$$
\left\{\begin{array}{l}
\sigma_{11} \\
\sigma_{22} \\
\sigma_{12}
\end{array}\right\}=T\left(\theta=45^{\circ}\right)^{-1}\left\{\begin{array}{c}
10 \\
0 \\
0
\end{array}\right\}
$$

so,

$$
\left\{\begin{array}{l}
\sigma_{11} \\
\sigma_{22} \\
\sigma_{12}
\end{array}\right\}=\left\{\begin{array}{c}
5 \\
5 \\
-5
\end{array}\right\} \mathrm{MPa},
$$

where $T$ is the matrix of transformation.

This plate was discretized using 4 continuous quadratic contour elements. Failure by the criterion of TsaiHill was analyzed in all the contour and an internal point in center of the lamina and the results are shown in Fig. 4. As can be seen, the numerical results showed that tension $\sigma_{11}$ 
for $\theta=45^{\circ}$ at every point of the contour and the internal point represent approximately the same value with smaller errors than $0.01 \%$ when compared with the analytical solution. It is possible also to note in Fig. 4 that the lamina presents shear deformations. This is due to load inclination in relation to the main reference material.

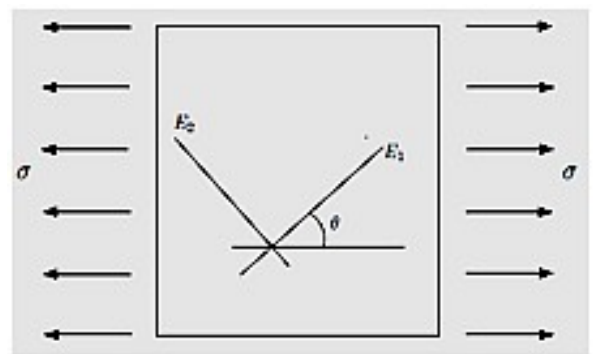

Fig. 3 Lamina square under uniform traction

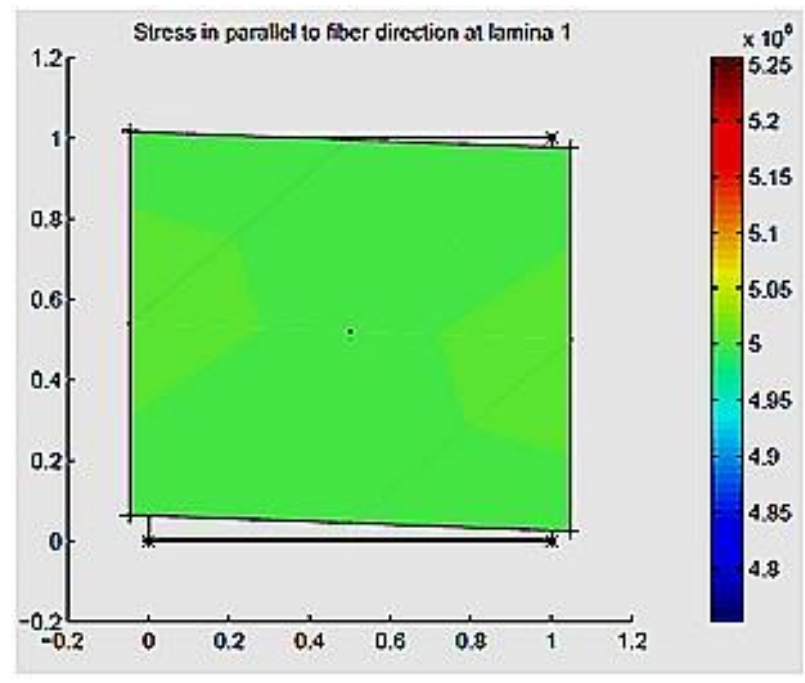

Fig. 4 Parallel stress fibers $\left(\sigma_{11}\right)$ for $\theta=45^{\circ}$

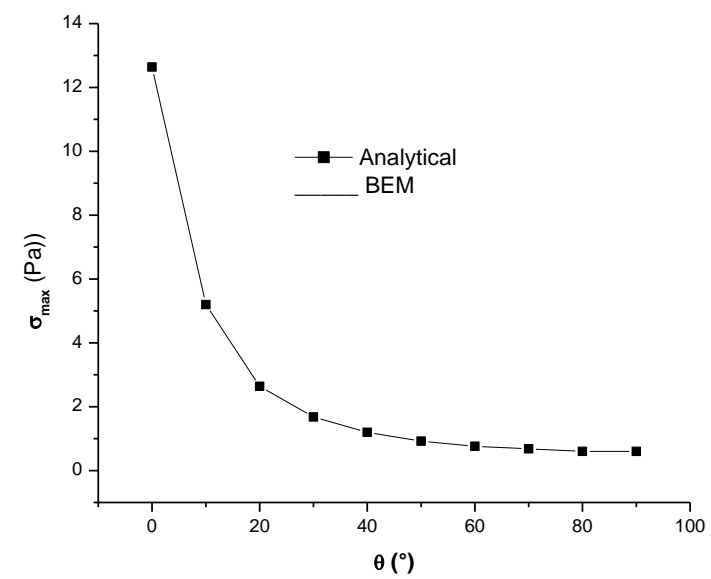

Fig. 5 Tensionwhat causes failure of the plate as a function of anglefiber orientation plate as a function of angle fiber orientation, according to the criterion of TsaiHill

To evaluate different configurations of the material, the angle was varied from zero to 90 and calculated the tension that causes the failure. The results of this study were compared with the analytical results presented by [33]. As can be noted in Fig. 5, there is a perfect concordance between the results numerical obtained in this work and the analytical results from the literature.

\subsection{Failure criteria for a lamina with hole}

Consider a lamina square of width $=1 \mathrm{~m}$ of an orthotropic material that has a radius hole $0,25 \mathrm{~m}$, and is under biaxial traction, as shown in Fig. 6. The material properties are: $E 1=14 \mathrm{GPa}, E 2=3,5 \mathrm{GPa}, G 12=4,2 \mathrm{GPa}, v 12=$ $=0,043$. The main axis of the material is oriented in an angle $\theta$ in relation to the $x$ axis. The properties of resistance of mate-rials are $X_{t}=1260 \mathrm{MPa}, Y_{t}=61 \mathrm{MPa}, X_{c}=500 \mathrm{MPa}$, $Y_{c}=102 \mathrm{MPa}$ and $S=167 \mathrm{MPa}$. At discretization of this lamina were used 46 quadratic continuous elements of con-tour, 28 elements being equal size on the outside edges of the lamina and 18 elements in the hole. It was calculated the tension $\sigma_{\max }$ to which the failed of lamina considering both

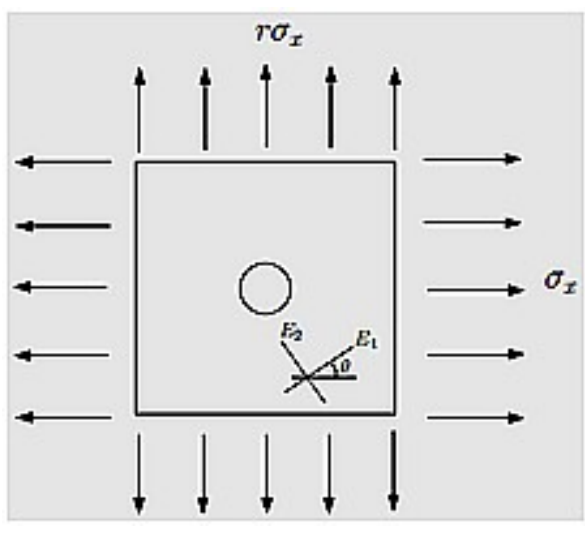

Fig. 6 Lamina subject to biaxial loading

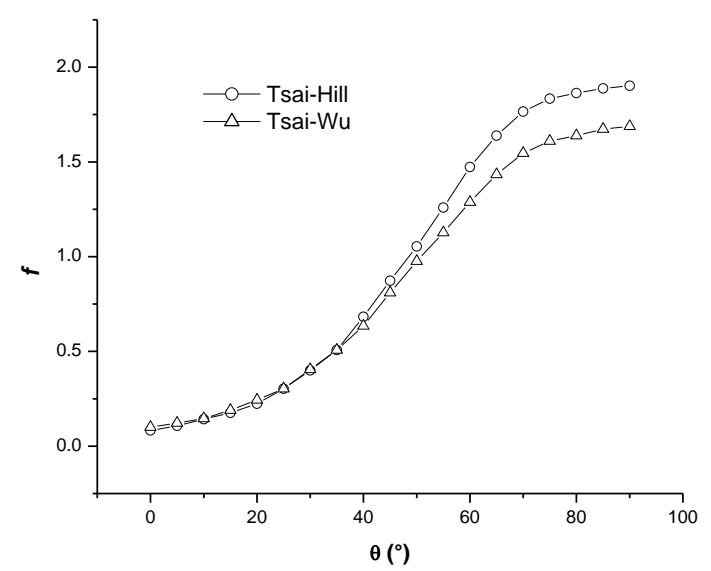

Fig. 7 Values of the failure criteria of Tsai-Hill and Tsai-Wu with $r=0$

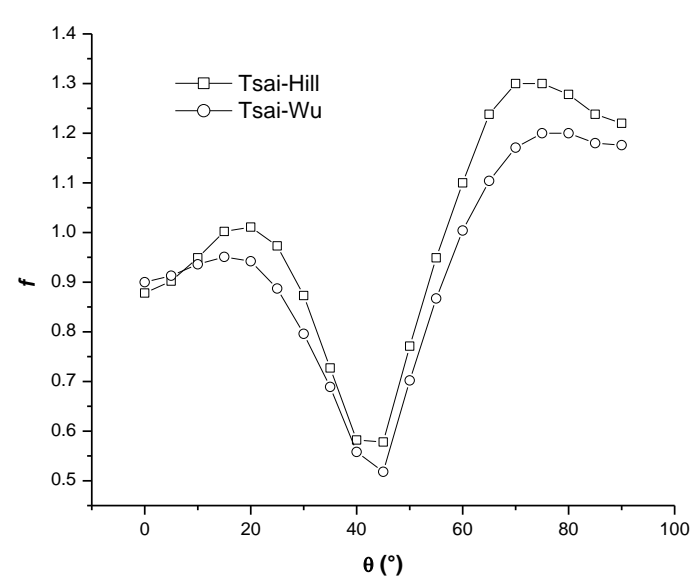

Fig. 8 Values of the failure criteria of Tsai-Hill and Tsai-Wu with $r=0.9$ 
the criterion of Tsai-Hill and the Tsai-Wu criterion. Figs. 7 and 8 show the stress $\sigma_{\max }$ that causes the failure of the lamina for $r=0$ and $r=0,9$ respectively. The criteria of TsaiHill and Tsai-Wu are compared. It can be noted that there is a good agreement on the angle of greater resistance (minimum value for $f$ ). However, there is a disagreement in the other regions which at some points is greater than $10 \%$.

\subsection{Failure criterion for laminate}

Consider a square lamina of width $a=1 \mathrm{~m}$ with a central hole of radius $r=0,25 \mathrm{~m}$ and thickness $T=4 \mathrm{~mm}$ in 4 layers of an orthotropic material that is under biaxial loading, as shown in Fig. 9. All layers are the same and have thicknesses of $1 \mathrm{~mm}$. The sequence of stacking the layers is $\left[\theta_{1} / \theta_{2}\right] \mathrm{s}$. The elastic properties of the materials are: $E 1=14$ $\mathrm{GPa}, E 2=3,5 \mathrm{GPa}, G 12=4,2 \mathrm{GPa}, v 12=0,043$. The properties of resistance of materials are $X_{t}=1260 \mathrm{MPa}, Y_{t}=$ $=61 \mathrm{MPa}, X_{c}=500 \mathrm{MPa}, Y_{c}=102 \mathrm{MPa}$ and $S=167 \mathrm{MPa}$. This lamina was discretized using 46 continuous quadratic elements of contour, and the edges 28 and the hole 18. Figs. 10 and 11 show the values of the Tsai-Hill failure criterion considering $\theta_{1}=0^{\circ}$ and $\theta_{2}=90^{\circ}$, respectively. To produce the color interpolation, the values of failure criteria were also calculated at some internal points. As can be noticed, the layers have different values for the failure criterion when the orientations of the fibers are different. In this example, the maximum value of $f(\theta)$ for layer $1\left(\theta_{1}=0^{\circ}\right)$, and $f 1=$ $=0,014$ and for Layer $2\left(\theta_{2}=90^{\circ}\right)$ and $f 2=0,627$. The value of the failure criterion of laminate is $f_{\max }=(f 1, f 2)=0.627$. And also noted that the maximum failure criterion occurs at the boundary, which confirms no need to calculate thefailure criteria in internal points as these will be not critical.

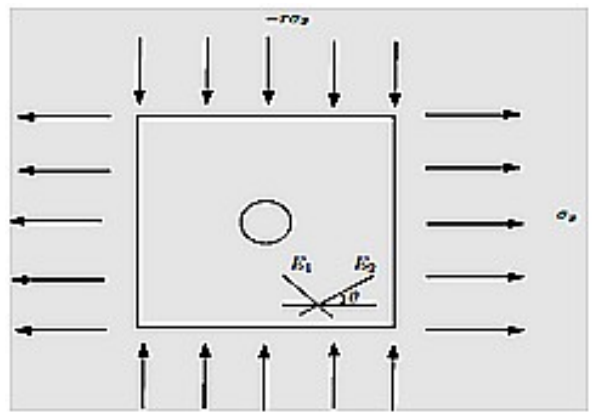

Fig. 9 Plate with central hole

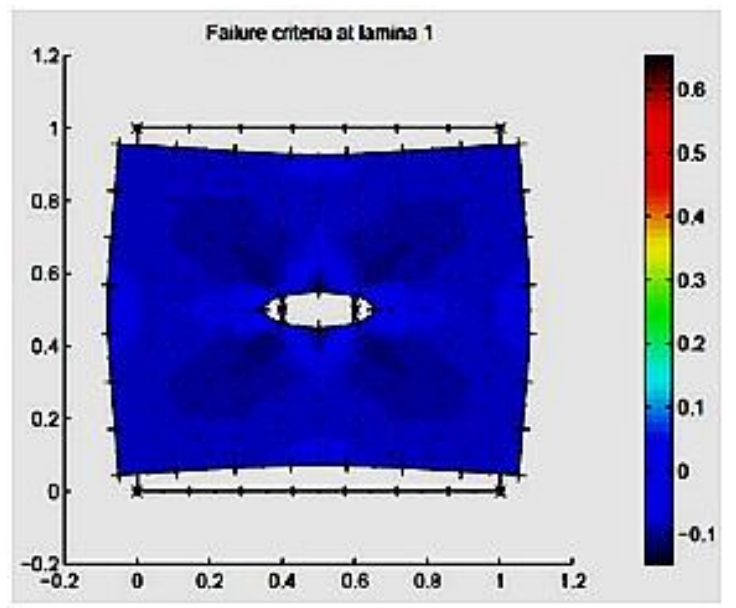

Fig. 10 Values for Tsai-Wu failure criterion for the first lamina $\left(\theta=0^{\circ}\right.$ and $\left.f_{\max }=0,014\right)$

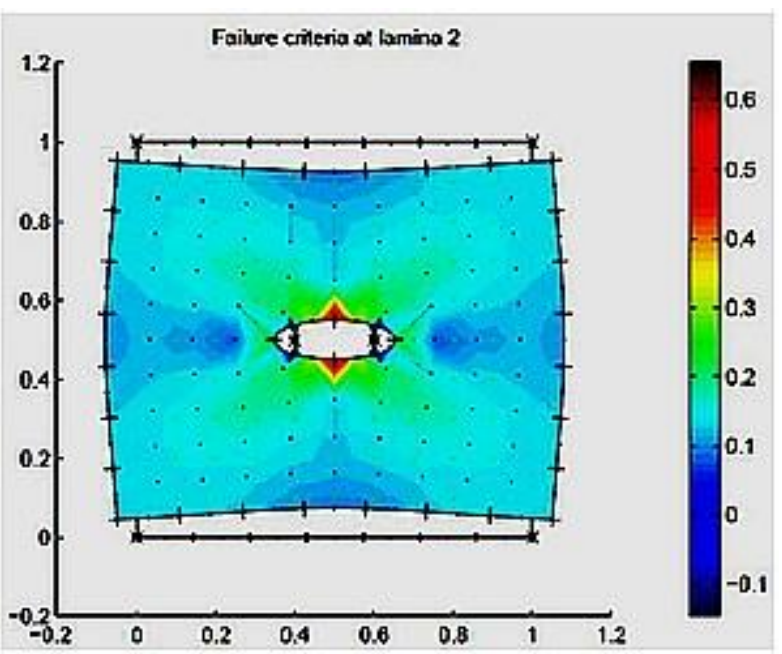

Fig. 11 Values for Tsai-Wu failure criterion for the second lamina $\left(\theta=90^{\circ}\right.$ and $\left.f_{\text {max }}=0,627\right)$

The Figs. 12 and 13 show the failure criteria of Tsai-Wu and Tsai-Hill, respectively, for $\theta_{1}$ and $\theta_{2}$ ranging between $90^{\circ}$ and $90^{\circ}$. It can be noted that the surfaces representing the failure criteria show differences significant. In addition, both surfaces have minimal local whose values are not identifiable unless you use some method of optimization. This difficulty in identifying the value of points minimum of failure criterion is higher largest for the number of blades that may have different orientations. The identification of these minimum points will be carried out in next section, where the methods of optimization are covered.

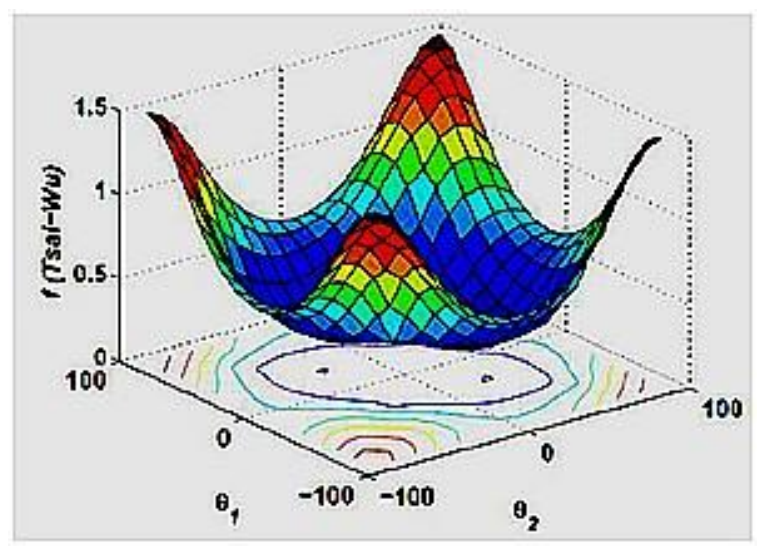

Fig. 12 Values for Tsai-Wu criterion

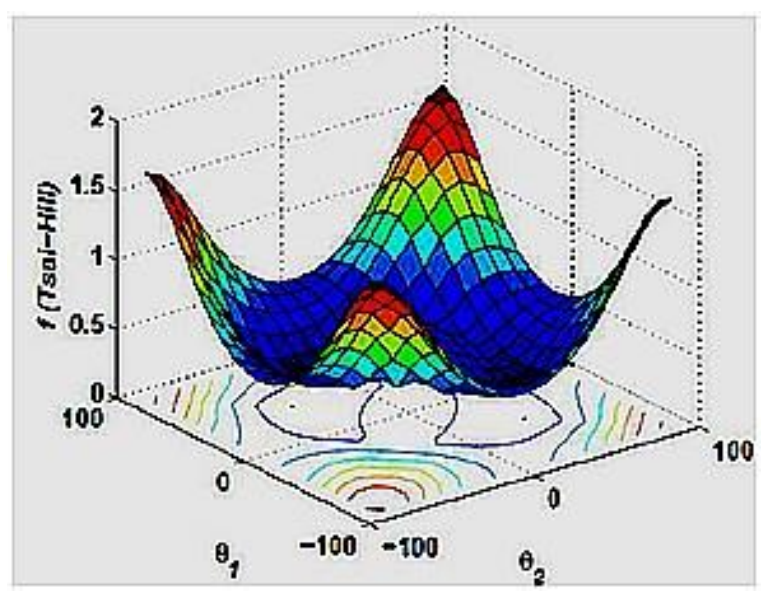

Fig. 13 Values for criterion of Tsai-Hill 


\section{For a lamina}

In order to evaluate the efficiency of the Golden Section method in optimization the direction of the fibers in a lamina with fibers arranged unidirectionally will be evaluated for two cases. The first case is lamina with hole under uniaxial traction (Fig. 14) and the second case is lamina with hole under biaxial traction (Fig. 6), both with the same data and using the criterion of Tsai-Hill. The values initial were $\theta_{1}=0^{\circ}$ and $\theta_{u}=90$. We obtained the convergence in case 1 after 14 iterations and they met as optimal orientation of the fibers the angle $\theta=0,0660^{\circ}$ (Fig. 15). In case 2 had the convergence after 13 iterations and found as the optimal orientation angle $\theta=42,6065^{\circ}$ (Fig. 16).

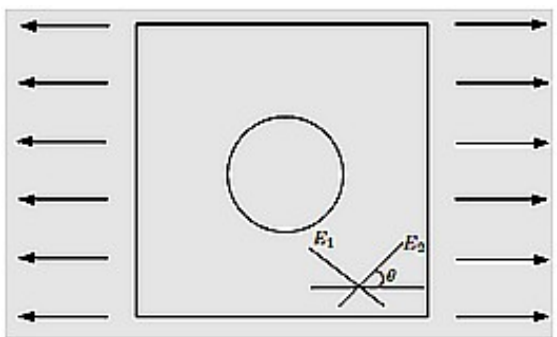

Fig. 14 Lamina under uniaxial traction.

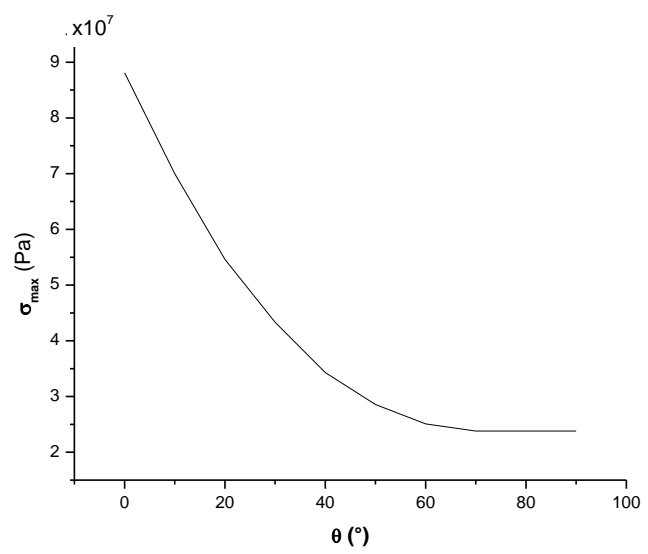

Fig. 15 Maximum stress that causes the failure

\section{For a laminate}

Consider the laminate shown in Fig. 9. With the goal to analyze the convergence of the modified simplex

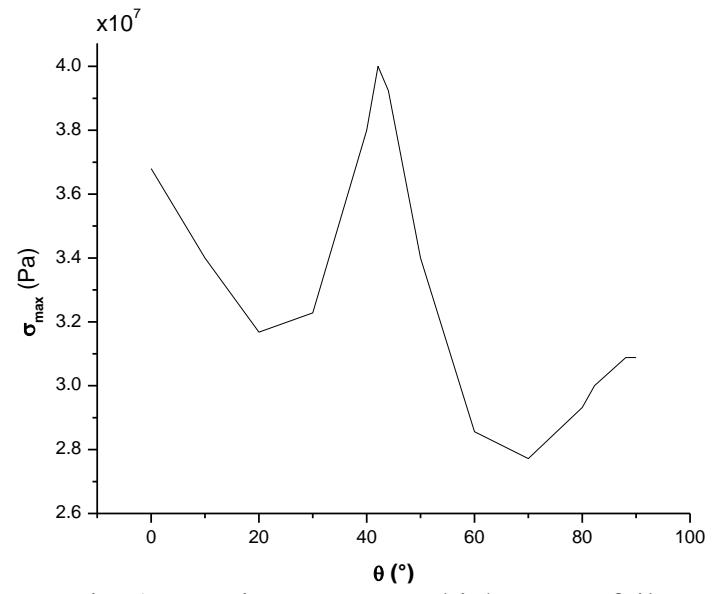

Fig. 16 Maximum stress which causes failure

method. The optimal values of the angles of the orientation of the fibers were calculated considering 7 cases (Table 1). In case 1 both the tolerance of $\mathrm{x}$ as the tolerance of the value of the function have small values, which provides a convergence to the minimum after a high number of iterations (55 and 53 for the criteria of Tsai-Hill and Tsai-Wu, respectively). In case the tolerance of $x 2$ has been increased, which has had no effect on the number of interactions, since the tolerance function of continued low. Increase in the tolerance of function in the case 3 and 4, there was a good lessening in number of iterations. In case 5 was varied the position of the starting point and convergence to the minimum occurred after the lowest number of interactions (16 and 18 for the criteria of Tsai-Hill and Tsai-Wu, respectively). In case 6 with the values of angles starting at $\theta_{1}=-70$ and $\theta_{2}=$ $=10$ is not converged to the correct value however, if diminished the value of the tolerance function the convergence occurs as shown in Case 7.

The analysis of the preceding paragraph concludes that the number of iterations is heavily dependent on tolerances and also the position of the starting point. However, excessively large tolerances can lead to wrong values of minimum. By the results obtained in this problem, it is suggested that Maximum values for the variables TolX and TolFun are 1 and $10^{-3}$ respectively. These values showed satisfactory results also for other laminates whose results are not shown in this paper.

Comparison between the criteria of Tsai-Hill and Tsai-Wu

Table 1

\begin{tabular}{|c|c|c|c|c|c|c|c|c|c|c|}
\hline & \multicolumn{4}{|c|}{ Input Values } & \multicolumn{3}{c|}{ Tsai-Hill } & \multicolumn{3}{c|}{ Tsai-Wu } \\
\hline Case & $\theta_{1}\left(^{\circ}\right)$ & $\theta_{2}\left(^{\circ}\right)$ & TolX & TolFun & $\begin{array}{l}\text { Number of } \\
\text { iterations }\end{array}$ & $\theta_{1}\left(^{\circ}\right)$ & $\theta_{2}\left(^{\circ}\right)$ & $\begin{array}{l}\text { Number of } \\
\text { iterations }\end{array}$ & $\theta_{1}\left({ }^{\circ}\right)$ & $\theta_{2}\left(^{\circ}\right)$ \\
\hline 1 & 70 & 10 & $10^{-1}$ & $10^{-5}$ & 55 & 46.7 & -46.7 & 53 & 44.4 & -44.4 \\
\hline 2 & 70 & 10 & 1 & $10^{-5}$ & 55 & 46.7 & -46.7 & 53 & 44.4 & -44.4 \\
\hline 3 & 70 & 10 & 1 & $10^{-3}$ & 40 & 46.9 & -46.5 & 32 & 44.5 & -44.5 \\
\hline 4 & 70 & 10 & 1 & $10^{-1}$ & 35 & 46.4 & -47 & 31 & 44.1 & -43.6 \\
\hline 5 & -70 & 70 & 1 & $10^{-1}$ & 16 & -46.7 & 46.8 & 18 & -44.5 & 43.8 \\
\hline 6 & -70 & 10 & 1 & $10^{-1}$ & 10 & -61.18 & 11.5 & 11 & -55.1 & 12.6 \\
\hline 7 & -70 & 10 & 1 & $10^{-3}$ & 44 & -46.8 & 46.4 & 40 & -44.2 & 44.4 \\
\hline
\end{tabular}

\section{Conclusion}

In this paper, it was presented a formulation of the method of boundary elements for the analysis of failure criteria in anisotropic materials submitted to efforts in the plan. They were used quadratic continuous contour elements in 
the discretization of laminas.

The values of the failure criteria were calculated in all the contour points in each of the laminas constituting the laminate. As the critical value of the failure criteria occurs at the boundary becomes unnecessary the use of internal points. It was shown that the main criteria failure (Tsai-Hill and Tsai-Wu) have small disagreements about values, depending on the material properties and boundary conditions, but in all cases analyzed there was a good agreement between the criteria as the many great angles for the laminate.

The optimization of methods used, which do not require the calculation of gradients, were suitable to the search of the optimal angles, converging after a few iterations. The objective function was given by the maximum value of the failure criterion, considering all the laminas.

\section{References}

1. Sollero, P.; Aliabadi, M.H. 1993. Fracture mechanics analysis of anisotropic plates by the boundary element method. Int. J. of Fracture 64: 269-284. https://link.springer.com/article/10.1007/BF00017845.

2. Deb, A.; Banerjee, P.K. 1990. BEM for general anisotropic 2D elasticity using particular integrals. Communications in Applied Numerical Methods 6(2): 111-119. http://onlineli-

brary.wiley.com/doi/10.1002/cnm.1630060207/full. http://dx.doi.org/10.1002/cnm.1630060207

3. Deb, A. 1996. Boundary elements analysis of anisotropic bodies under thermo mechanical body force loadings. Computers and Structures 58: 715-726. http://dx.dx.doi.org/10.1016/0045-7949(95)00196-N.

4. Albuquerque, E. L.; Sollero, P. 1998. The boundary element method applied to transient dynamic anisotropic problems. In Kassab, A. J., Brebbia, C. A., e Chopra, M., editors, Proc. Boundary Element Method XX, Orlando USA: 617-624,.

5. Kogl, M;. Gaul, L. 2000. A boundary element method for transient piezoelectric analysis. Engn. Anal. with Boundary Elements 24: 591-598. http://dx.doi.org/10.1016/S0955-7997(00)00039-4.

6. Albuquerque, E.L.; Sollero, P.; Aliabadi, M.H. 2002. The boundary element method applied to time dependent problems in anisotropic materials. International Journal of Solids and Structure 39: 1405-1422. http://dx.doi.org/10.1016/S0020-7683(01)00173-1.

7. Albuquerque, E.L.; Sollero, P.; Fedelink, P. 2003. Dual reciprocity boundary element method in Laplace domain applied to anisotropic dynamic crack problems. Computers and Structures 81: 1707-1713. http://dx.doi.org/10.1016/S0045-7949(03)00184-6.

8. Albuquerque, E.L.; Sollero, P.; Fedelink, P. 2003. Free vibration analysis of anisotropic material structures using the boundary element method. Engineering Analysis with boundary element 27: 977-985. http://dx.doi.org/10.1016/S0955-7997(03)00074-2.

9. Albuquerque, E.L.; Sollero, P.;Aliabadi, M.H. 2004. Dual boundary element method for anisotropic dynamic fracture mechanics. International Journal for Numerical Methods in Engineering 59: 1187-1205.

http://onlinelibrary.wiley.com/doi/10.1002/nme.912/full.

10. Bendsøe, M.P.; Kikuchi, N. 1988. Generating optimal topologies in structural design using a homogenization method. Comp. Meth. Appl. Mech. Engrg. 71: 197-224. http://dx.doi.org/10.1016/0045-7825(88)90086-2.

11. Zhou, M.; Rozvany, G.I.N. 1991. The COC algorithm: Part II: topological, geometrical and generalized shape optimization. Comp. Meth. Appl. Mech. Engrg. 89: 309336.

http://dx.doi.org/10.1016/0045-7825(91)90046-9.

12. Rozvany, G.I.N.; Bendsøe, M.P.; Kirsch, U. 1995. Layout optimization of structures. Appl. Mech. Rev. 48: 41-119. http://dx.doi.org/10.1115/1.3005097.

13. Bendsøe, M.P.; Sigmund O. 2003. Topology Optimization - Theory, Methods and Applications. Springer, Berlin.

14. Zhou, M.; Pagaldipti, N.; Thomas, H.L.; Shyy Y.K. 2004. An Integrated Approach for Topology, Sizing, and Shape Optimization. Struct. Multidisc. Optim. 26: 308317.

https://link.springer.com/article/10.1007/s00158-0030351-2.

15. Cervellera, P.; Zhou, M.; Schramm, U. 2005. Optimization driven design of shell structures under stiffness, strength and stability requirements. Proc. 6th World Congresses of Structural and Multidisciplinary Optimization, Rio de Janeiro, Brazil.

16. Schramm, U.; Zhou, M.; Tang, P.S.; Harte, C.G. 2004. Topology Layout of Structural Designs and Buckling, AIAA-2004-4636, Proc. 10th AIAA/ISSMO Symposium on Multidisciplinary Analysis and Optimization, Albany, NY.

17. Krog, L.; Tucker, A.; Rollema, G. 2002. Application of Topology, Sizing and Shape Optimization Methods to Optimal Design of Aircraft Components, Proc. 3rd Altair UK HyperWorks Users Conference.

18. Krog, L.; Tucker, A.; Kempt, M.; Boyd, R. 2004. Topology Optimization of Aircraft Wing Box Ribs, AIAA2004-4481, Proc. 10th AIAA/ISSMO Symposium on Multidisciplinary Analysis and Optimization, Albany, NY.

19. Zhou, M.; Fluey, R.; Willmet, T. 2007. Multiple phase optimization of composite structures. Proc. 9th US National Congress of Computational Mechanics, San Francisco, July.

20. Zhou, M.; Fluery, R.; Dias, W. 2009. Composite design optimization - from concept to ply-book details. Proc. 8th World Congress of Structural and Multidisciplinary Optimization, Lisbon, Portugal.

21. Altair OptiStruct Version 10.0, Users manual. Altair Engineering, Inc., Troy, MI, 2009.

22. Farshi, B.; Herasati, S. 2005. Optimum weight design of fiber composite plates in flexure based on a two level strategy. Composites Science and Technology 58: 571589. http://dx.doi.org/10.1016/j.compstruct.2005.03.002.

23. Hill, R. 1950. A Theory of the Yelding and Plastic Flow of Anisotropic Metals," Proceedings of the Royal Socety 193: 281-297. http://dx.doi.org/10.1098/rspa.1948.0045.

24. Tsai, S.W. 1968. Strength Theories of Filamentary Structures, in Fundamental Aspects of Fiber Reinforced Plastic Composites, R.T. Schwartz and H.T. Schwartz Eds., Wiley Interscience, New York, US, pp. 3-11.

25. Tsai, S.W.; Wu, E.M. 1971. A General Theory of Strength Test for Anisotropic Materials, Journal of 
Composite Materials 5: 58-80.

http://jour-

nals.sagepub.com/doi/abs/10.1177/0021998371005001 06.

26. Lekhnitskii, S.G. 1963. Theory of Elasticity of an Anisotropic Elastic Body. Holden-Day: San Francisco.

27. Lekhnitskii, S.G. 1968. Anisotropic plates. Gordon and Breach, New York.

28. Fedelinski, P.; Aliabadi, M.H.; Rooke, D.P. 1993. The dual boundary element method in dynamic fracture mechanics. Engineering Analysis with Boundary Elements 12: $203-210$. http://dx.doi.org/10.1016/0955-7997(93)90016-E.

29. Sollero, P. 1994. Fracture mechanics analysis of anisotropic laminates by the boundary element method. Ph.D. Thesis, Wessex Institute of Technology.

30. Haftka, R. T.; Gurdal, Z. 1991. Elements of structural optimization. Dordrech, Kluwer, third edition.

31. Bazarra, M.S.; Sherali, H.D.; Shetty, C.M. 1993. Nonlinear Programming - theory and algorithms. John Wiley and Sons Inc., New York.

32. Vanderplaats, G.N.1999. Numerical optimization techniques for engineering design. Vanderplaats Research and Development, Colorado Springs, 3rd edition.

33. Gurdal, Z.; Haftka, R.T.; Hajela, P. 1998. Design and optimization of laminated composite materials. John Wiley and Sons Inc., New York, 1998.
S. Debbaghi, A. Sahli, Sara Sahli

\section{OPTIMIZATION AND FAILURE CRITERIA FOR COMPOSITEMATERIALS BY THE BOUNDARY ELEMENT METHOD}

S u m m a r y

The present paper deals with the analysis of the main failure, considering two quadratic criteria: the TsaiHill criterion and the Tsai-Wu criterion, for composite materials using the method of boundary elements. The developed formulation is applied to compute the stress and displacements of laminated composite structures plain submitted to loads in the structure plane, to show the influence of the direction of fibre in failure of the first lamina in a laminated composite materials, and the value of maximum tension that causes the failure, to find the optimum orientation of the directions of fibers into a symmetrical laminate, to minimize the failure criteria of Tsai-Hill and Tsai-Wu, and to optimum values for the fibers orientation angles that maximize the strength of the structure. The results are compared with other results from literature and show a good agreement.

Keywords: failure criteria; composite materials; boundary elements method; Optimization; fibers orientation.

Received April 30, 2016

Accepted August 04, 2017 